\title{
A TRAVELING WAVE TERMINATION FOR A THERMOACOUSTIC SETUP
}

\author{
M.C. Vidya*, J.P. Oosterhuis, and T.H. van der Meer \\ Thermal Engineering, Engineering Technology, University of Twente, P.O. Box 217, 7500AE, Enschede, The Netherlands. \\ ${ }^{*}$ Corresponding author's e-mail: m.c.vidya@utwente.nl
}

Keywords: traveling wave, impedance tube, reflection coefficient, sound absorption.

\section{Introduction}

A modification of a thermoacoustic experimental setup is performed to obtain traveling wave conditions. The setup has been used previously at the University of Twente to conduct experiments in a standing wave environment. One method to obtain a traveling wave inside the setup is to use a sound absorbing device. Two classes of sound absorbing structures can be distinguished: porous materials and resonance absorbers [4]. A traveling wave termination in the form of a resonance absorber was designed and tested.

\section{Method}

The experimental setup is depicted in Figure 1. For a detailed description, readers are referred to $[1,5]$. The termination of the main impedance tube can be removed, thus a traveling wave termination can be attached to the end of the setup at $x=0$. The termination is made of a quarter-wavelength tube with a diameter such that the wave is dissipated.

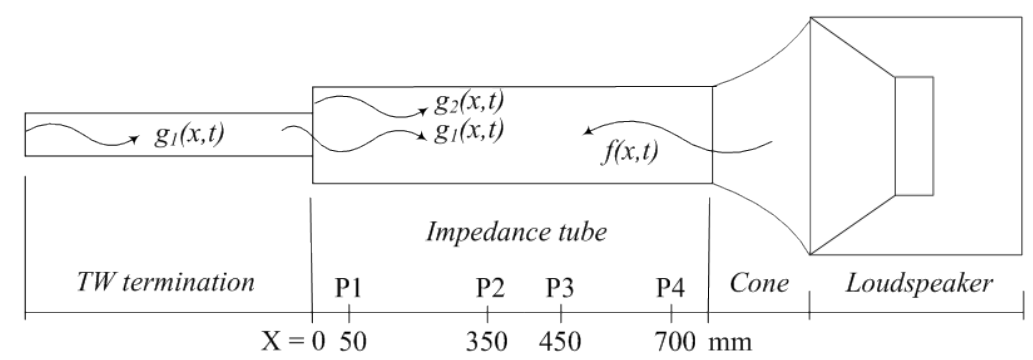

Figure 1: Experimental setup with attached traveling wave termination. P1 - P4 represents the location of pressure sensors. Figure not to scale.

The idea of adding a traveling wave termination is to cancel the reflections at the end of the setup. When an incoming wave $f(x, t)$ is generated from the loudspeaker, it will be reflected at the end of the setup (wave $g_{2}(x, t)$ ). The incoming wave $f(x, t)$ will also be reflected by the end wall of the traveling wave termination (wave $g_{1}(x, t)$ ). Wave $g_{2}(x, t)$ should have equal amplitude but $180^{\circ}$ phase difference to $g_{1}(x, t)$ such that all reflected waves cancel each other at $x=0$.

To model the wave propagation inside the impedance tube and the traveling wave termination, a one dimensional Low Reduced Frequency Model [3] is used. From the solution of the Low Reduced Frequency Model, the reflection coefficient and subsequently, absorption coefficient can be obtained as follows,

$$
R(x)=\frac{\hat{p}_{A} e^{\Gamma k x}}{\hat{p}_{B} e^{-\Gamma k x}} \quad \alpha(x)=1-|R|^{2}
$$


where $\hat{p}_{A}$ and $\hat{p}_{B}$ are the complex pressure amplitudes of the backward and forward propagating wave, respectively.

\section{Results}

The one dimensional model was used to obtain the relation between tube radius, tube length, absorption coefficient, and frequency. For each tube radius, there is one frequency and tube length that correspond to the theoretical $100 \%$ absorption. The manufactured tube has an inner radius of $5.985 \mathrm{~mm}$ and length of $73.7 \mathrm{~cm}$. According to the model, it has a theoretical absorption coefficient of $100 \%$ at a frequency of $113 \mathrm{~Hz}$.

Once the traveling wave termination was built, two sets of experiments were conducted: (1) a frequency sweep at pressure amplitude of $100 \mathrm{~Pa}$ and (2) a pressure amplitude sweep at 113 Hz. The results are presented in Figure 2.
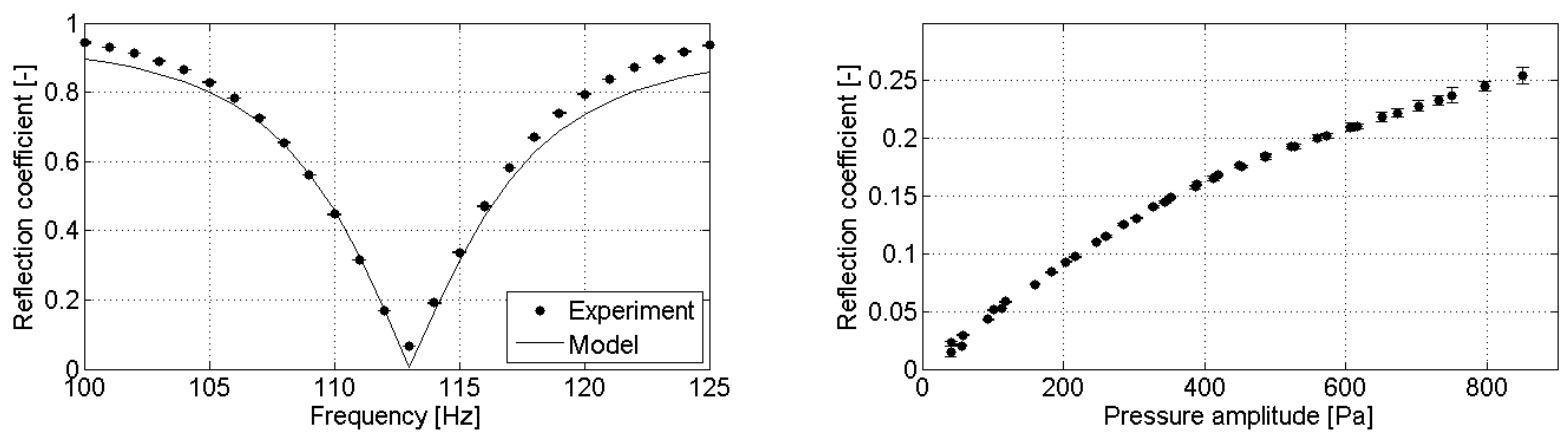

Figure 2: Experimental results of frequency sweep at P2 = $100 \mathrm{~Pa}$ (left) and pressure amplitude sweep at $113 \mathrm{~Hz}$ (right). Error bars represent standard deviation within one measurement point.

The traveling wave termination works well at low amplitudes and at the predicted frequency. At higher pressure amplitude, the reflection coefficient increases nonlinearly. Possible causes for this phenomenon are tube imperfection or nonlinear effects $[1,2]$ that occur at high pressure amplitude or due to flow disruption at $x=0$.

\section{Conclusions}

A standing wave thermoacoustic setup is converted into a traveling wave setup by using a traveling wave termination. The termination works best at $113 \mathrm{~Hz}$ with absorption of $99.9 \%$.

\section{Acknowledgements}

The authors would like to gratefully thank Jos Zeegers and the Eindhoven University of Technology for the provision of the experimental apparatus. Bosch Thermotechnology and Agentschap NL are thankfully acknowledged for the financial support as part of the EOSKTO research program under project number KTOT03009.

\section{References}

[1] Aben, P. C. H. High-Amplitude Thermoacoustic Flow Interacting with Solid Boundaries. Phd thesis, Technische Universiteit Eindhoven (2010).

[2] in 't Pahuis, P. H. M. W. Mathematical aspects of thermoacoustics. Phd thesis, Technische Universiteit Eindhoven (2009).

[3] Tijdeman, H. “On the Propagation of Sound Waves in Cylindrical Tubes". J. Sound and Vibration, 39(1), (1975), $1-33$.

[4] van der Eerden, F. J. M. Noise Reduction with Coupled Prismatic Tubes. Phd thesis, University of Twente (2000).

[5] van der Gun, D. Design and Realization of an Experimental Thermoacoustic Setup. Master thesis, University of Twente (2013). 